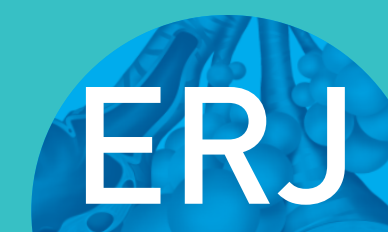

open research
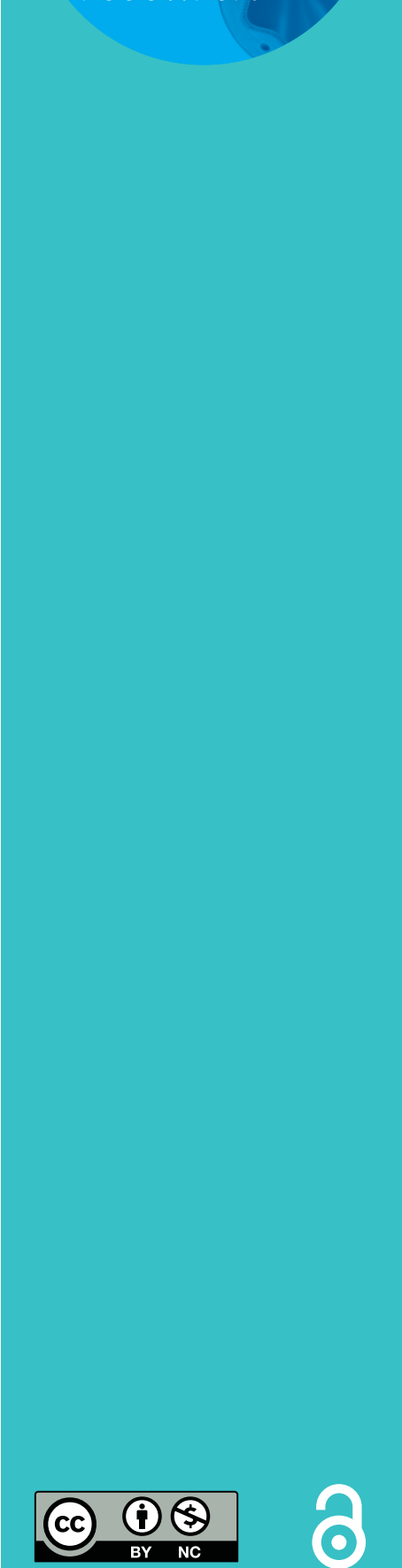

\title{
MRSA eradication of newly acquired lower respiratory tract infection in cystic fibrosis
}

\author{
Emilie Vallières ${ }^{1,2}$, Jacqueline C. Rendall ${ }^{1,3}$, John E. Moore $2,4,5$, \\ John McCaughan ${ }^{3,4}$, Anne I. Hoeritzauer ${ }^{6}$, Michael M. Tunney ${ }^{1,7}$, \\ Joseph Stuart Elborn ${ }^{1,2,3}$ and Damian G. Downey ${ }^{1,3}$
}

\begin{abstract}
Affiliations: ${ }^{1}$ Cystic Fibrosis \& Airways Microbiology Research Group, Queen's University Belfast, Belfast, UK ${ }^{2}$ Centre for Infection and Immunity, Queen's University Belfast, Belfast, UK. ${ }^{3}$ Northern Ireland Regional Adult CF Centre, Belfast, UK. ${ }^{4}$ Dept of Bacteriology, Belfast Health and Social Care Trust, Belfast, UK. ${ }^{5}$ School of Biomedical Sciences, Ulster University, Coleraine, UK. ${ }^{6}$ Dept of Neurosciences, University of Edinburgh, Western General Hospital, Edinburgh, UK. ${ }^{7}$ School of Pharmacy, Queen's University Belfast, Belfast, UK.
\end{abstract}

Correspondence: Emilie Vallières, Centre for Infection and Immunity, Health Sciences Building, 97 Lisburn Road, Belfast, BT9 7BL, UK. E-mail: evallieres01dqub.ac.uk

ABSTRACT UK cystic fibrosis (CF) guidelines recommend eradication of methicillin-resistant Staphylococcus aureus (MRSA) when cultured from respiratory samples. As there is no clear consensus as to which eradication regimen is most effective, we determined the efficacy of eradication regimens used in our CF centre and long-term clinical outcome.

All new MRSA positive sputum cultures $(n=37)$ that occurred between 2000 and 2014 were reviewed. Eradication regimen characteristics and clinical, microbiological and long-term outcome data were collected.

Rifampicin plus fusidic acid was the most frequently used regimen (24 (65\%) out of 37 patients), with an overall success rate of $79 \%$ (19 out of 24 patients). Eradication failure was more likely in patients with an additional MRSA-positive peripheral screening swab $(\mathrm{p}=0.03)$ and was associated with worse survival $(\mathrm{p}=0.04)$.

Our results demonstrate the feasibility and clinical benefits of MRSA eradication. As peripheral colonisation was associated with lower eradication success, strategies combining systemic and topical treatments should be considered to optimise outcomes in CF patients.

@ERSpublications

In CF, eradication of MRSA from the respiratory tract is feasible and is associated with better clinical outcome http://ow.ly/YfbqD

Received: Sept 012015 | Accepted after revision: Jan 292016

Support statement: E. Vallières was funded by an AMMI Canada/Pfizer Fellowship and grants from Cystic Fibrosis Canada Fellowship.

Conflict of interest: Disclosures can be found alongside this article at openres.ersjournals.com

The content of this work is @ the authors or their employers. Design and branding are @ERS 2016. This article is open access and distributed under the terms of the Creative Commons Attribution Non-Commercial Licence 4.0. 


\section{Introduction}

Methicillin-resistant Staphylococcus aureus (MRSA) is a clinically significant pathogen in cystic fibrosis (CF) pulmonary infection. Large cohort studies indicate that chronic respiratory infection with MRSA is associated with a faster decline in lung function amongst children [1-4] and worse survival in the adult population [5]. Therefore, current guidelines recommend eradication of MRSA when first isolated in respiratory samples from CF patients $[6,7]$.

The UK CF Trust guidelines recommend that a combination of rifampicin and fusidic acid should be used, with or without inhaled vancomycin, for MRSA airways eradication [6]. However, there is little evidence to support this recommendation. In fact, the optimal eradication regimen to treat CF MRSA respiratory infection is currently disputed, with a number of ongoing clinical trials investigating chronic MRSA eradication strategies [8]. Recent studies have reported small case series, describing a range of eradication strategies $[9,10]$. The duration of eradication regimens described also varies from a few days to several months [11-13], using either a single agent or a combination of oral and/or intravenous antibiotics $[14,15]$. Inhaled vancomycin was used by some groups but without definitive or conclusive results [16]. Furthermore, the characteristics of the CF populations included were diverse, with some studies investigating acute MRSA infections only and others incorporating chronically infected patients into their analyses [13]. A recent Cochrane review found that there is insufficient data to recommend a specific MRSA eradication regimen, and that prospective studies are warranted [17].

In order to optimise and standardise our current practice, we undertook a retrospective study to determine the efficacy of the different eradication regimens used in our centre over the past 15 years. Between 2000 and 2014, the Northern Ireland Regional Adult CF Centre (Belfast, UK) patient cohort has steadily increased, from approximately 150-294 patients. During this time, it was part of our policy to eradicate new MRSA as soon as it was detected in a respiratory sample culture, without awaiting a second confirmatory sample. However, no official consensus eradication regimen was established, including selection of antibiotics and treatment duration. These decisions were made on an individual basis according to patient medical history (e.g. allergies and liver disease), antimicrobial susceptibility testing results and clinician preference. As recommended by CF and non-CF specific guidelines [18-20], 2\% nasal mupirocin ointment and $4 \%$ chlorhexidine body wash for 5 days were routinely prescribed, in addition to systemic antibiotics. Inhaled vancomycin was not used in our centre as part of an eradication regimen.

\section{Materials and methods}

Study design

Using the clinical microbiology database and the CF registry, we identified patients attending the Northern Ireland Regional Adult CF Centre who acquired a new MRSA respiratory infection between January 1, 2000 and December 31, 2014. The medical charts of these patients were reviewed to collect relevant clinical, microbiology and long-term outcome data. This case series study was registered as an audit in the Belfast Health and Social Care Trust.

\section{Definitions}

A new MRSA episode was defined as at least one positive respiratory sample culture (sputum or oropharyngeal swab) from a person whose previous respiratory cultures (during the previous 12 months) were negative for MRSA. MRSA was considered successfully eradicated if all respiratory samples, during the 6-month period following eradication treatment, were MRSA culture negative. A minimum of two cultures, collected after the end of systemic antibiotic treatment, were required to fulfil the eradication criteria. Patients who had at least two MRSA-positive sputum cultures over a 6-month period prior to the first eradication attempt were defined as chronically infected with MRSA.

\section{Statistical analyses}

We conducted a descriptive analysis of the antibiotic regimens used and the population treated, based on data collected at the start of eradication treatment. Continuous variables are expressed as a mean $\pm \mathrm{SD}$, whilst categorical variables were expressed using proportions or percentages. Bivariate analyses were conducted using the Chi-squared or Fisher's exact test where appropriate. Continuous variables were compared using a t-test or Mann-Whitney test for non-parametric data. Long-term outcomes were described using Kaplan-Meier survival curves and differences assessed using the log-rank test. A p-value $\leqslant 0.05$ was considered statistically significant in all analyses.

\section{Results}

\section{Description of MRSA episodes}

During the study period 38 new MRSA episodes were identified, with a median (range) of 2.5 (0-6) cases.year ${ }^{-1}$. Two patients had two distinct episodes that occurred $>3-5$ years after their first eradication trial. 
One patient spontaneously cleared MRSA infection without any specific eradication treatment. This case was excluded from subsequent analysis, leaving 37 eligible episodes. 36 (97\%) out of 37 MRSA episodes were acute with the remaining case considered a chronic infection (duration of 33 weeks). For this latter case, poor attendance at clinic appointments precluded earlier initiation of eradication treatment.

\section{Description of eradication regimens used}

Eradication regimens were defined by both the antibiotics chosen and the duration of treatment. According to these criteria, nine different eradication regimens were used during the study period. As shown in table 1 , 42 -day treatment with rifampicin and fusidic acid was the most frequent regimen prescribed (20 (54\%) out of 37 patients). This dual therapy was given in four additional cases for a shorter course of 3 weeks. Monotherapy with doxycycline was used on six occasions, whilst the combination of minocycline with fusidic acid was prescribed a total of three times. The four remaining regimens were used once.

The overall success rate of the eradication regimens prescribed was $73 \%$ (27 out of 37 patients). The combination of rifampicin with fusidic acid (3- and 6-week courses) was most effective with successful eradication achieved in 19 (79\%) out of 24 identified cases (table 2). Monotherapy with doxycycline was effective for MRSA eradication in four (66\%) out of six cases (table 2). The infrequent use of the remaining regimens precludes determination of their efficacy. Due to gastrointestinal intolerance, two patients treated with rifampicin and fusidic acid had their eradication regimen modified while under treatment.

\section{Impact of eradication regimens}

To determine the effect of treatment duration on eradication, we compared the success rate of all the regimens given for $\leqslant 3$ weeks (short regimens: $n=8$ ) with those given for $>3$ weeks (long regimen: $n=29$ ) (table 2). There was no statistically significant difference in efficacy ( $\mathrm{p}=0.40$, Fisher's exact test).

We also evaluated whether combination therapy with two antibiotics was more effective than monotherapy. Amongst eradication failures, four (40\%) out of 10 were associated with single agent treatments. Furthermore, less than one-fifth of the successful eradications (five (18.5\%) out of 27) were achieved using monotherapy. However, these differences were not statistically significant ( $p=0.22$, Fisher's exact test).

\section{Patient characteristics and long-term outcomes}

To identify clinical characteristics that might impact on eradication outcome, we grouped patients as either eradication success $(n=27)$ or eradication failure $(n=10)$ and compared a range of parameters (table 3 ). There was no statistically significant difference between groups with regard to sex and age distribution, forced expiratory volume in $1 \mathrm{~s}(\mathrm{FEV} 1)$ values, prevalence of pancreatic insufficiency and CF-related diabetes, Pseudomonas aeruginosa infection status and duration of MRSA infection prior to initiation of eradication. Although the genotype profile was statistically similar between both groups ( $\mathrm{p}=0.5$, Chi-squared test), there was a higher proportion of F508del-homozygous patients amongst the MRSA eradication failure group (six (60\%) out of 10) compared to the MRSA eradication success group (10 (37\%) out of 27). Furthermore, eradication failure was more likely in patients who had at least one positive peripheral screening swab (nasal and/or skin) ( $\mathrm{p}=0.03$, Fisher's exact test).

Chronic infection with MRSA is associated with a poorer prognosis [1,5]. Therefore, we compared long-term clinical outcomes in the two groups (eradication success versus failure). Difference in FEV 1 (measured 1 year after initiation of eradication) was not different between both groups. Using Kaplan-Meier

\section{TABLE 1 Use and duration of treatment}

Treatment
Rifampicin and fusidic acid
Doxycycline
Minocycline and fusidic acid
Rifampicin and TMP
Clindamycin
Teicoplanin
TMP-SMX
Total

7 days 14 days

\section{Regimen 21 days}

4

3

\section{1}

1

\section{8 days}

Data are presented as n. TMP: trimethoprim; TMP-SMX: trimethoprim-sulfamethoxazole. 
TABLE 2 Success rate of individual eradication regimens

Treatment
Rifampicin and fusidic acid
Doxycycline
Minocycline and fusidic acid
Rifampicin and TMP
Clindamycin
Teicoplanin
TMP-SMX

TMP-SMX

\begin{tabular}{cc}
\multicolumn{2}{c}{ Regimen } \\
s $^{\text { weeks }}{ }^{\#}$ & $>3$ weeks \\
$4 / 4(100)$ & $15 / 20(75)$ \\
$2 / 3(66)$ & $2 / 3(66)$ \\
NA & $2 / 3(66)$ \\
NA & $1 / 1(100)$ \\
NA & $0 / 1(0)$ \\
$1 / 1(100)$ & $N A$ \\
NA & $0 / 1(0)$
\end{tabular}

Data are presented as $\mathrm{n} / \mathrm{N}(\%)$. TMP applicable. ${ }^{\#}: \mathrm{n}=8 ;{ }^{\text {I }}: \mathrm{n}=29 ;{ }^{+}: \mathrm{n}=37$

survival curves and log-rank statistical analyses (figure 1), no significant difference was detected in the time elapsed between eradication and the next pulmonary exacerbation requiring intravenous antibiotics. However, the survival rate was significantly worse amongst the MRSA eradication failure group (four deaths) compared to those with a successful eradication (two deaths) $(\mathrm{p}=0.04)$.

\section{Discussion}

Over the study period (2000-2014), the incidence of MRSA infection in our CF population remained low, with an average of 2.5 new respiratory infections per year. In this study, we reviewed 37 new MRSA episodes in which an eradication regimen was prescribed and this represents, to our knowledge, the largest case series investigating eradication of acute MRSA infection. In accordance with current UK guidelines [6], rifampicin combined with fusidic acid was the preferred regimen in our CF centre, with a success rate of $79 \%$, which is comparable with the results of previous studies where efficacy of eradication treatments was approximately $80 \%[10,12,14]$.

Treatment burden and side-effects are major concerns in the CF population. The combination of rifampicin and fusidic acid for MRSA airways eradication is frequently associated with gastrointestinal side-effects [13]. Several week courses with these drugs also necessitate close liver function monitoring and therapy may not be well tolerated, leading to discontinuation. Two patients treated with rifampicin and fusidic acid experienced gastrointestinal symptoms that led to a modification of their eradication regimen. No significant changes in hepatic enzymes were noted. Taking into account the high incidence of side-effects related to these drugs and the fact that there is no specific duration of treatment recommended in the current guidelines [6], we compared the efficacy of shorter regimens with those lasting $>3$ weeks (table 2). Although strong conclusions are limited by the small number of patients who received a short

\begin{tabular}{|c|c|c|c|}
\hline & Success & Failure & p-value ${ }^{+}$ \\
\hline Subjects & 27 & 10 & \\
\hline Male/female & $17 / 10$ & $5 / 5$ & 0.71 \\
\hline Age years & $30.9 \pm 17.2$ & $28.4 \pm 9.5$ & 0.67 \\
\hline \multicolumn{3}{|l|}{ Genotype } & \multirow[t]{4}{*}{0.50} \\
\hline F508del homozygous & 10 (37) & $6(60)$ & \\
\hline F508del heterozygous & $15(55.6)$ & $4(40)$ & \\
\hline Other & $2(7.4)$ & 0 & \\
\hline Pancreatic insufficiency & $21(77.8)$ & $9(90)$ & 0.65 \\
\hline CF-related diabetes & $6(22.2)$ & $3(30)$ & 0.68 \\
\hline FEV $1 \%$ predicted & $66.9 \pm 24.1$ & $61.5 \pm 18.2$ & 0.57 \\
\hline Pseudomonas aeruginosa" & 22 (81.5) & $8(80)$ & 0.92 \\
\hline Duration of infection weeks $s^{\text {I }}$ & $3.7 \pm 6.6$ & $2.6 \pm 3.1$ & 0.60 \\
\hline MRSA carrier nasal/skin swabs & $4(14.8)$ & $6(60)$ & 0.03 \\
\hline \multicolumn{4}{|c|}{ 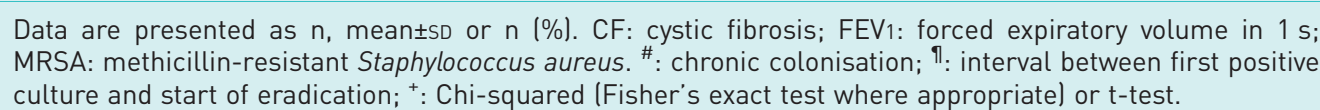 } \\
\hline
\end{tabular}



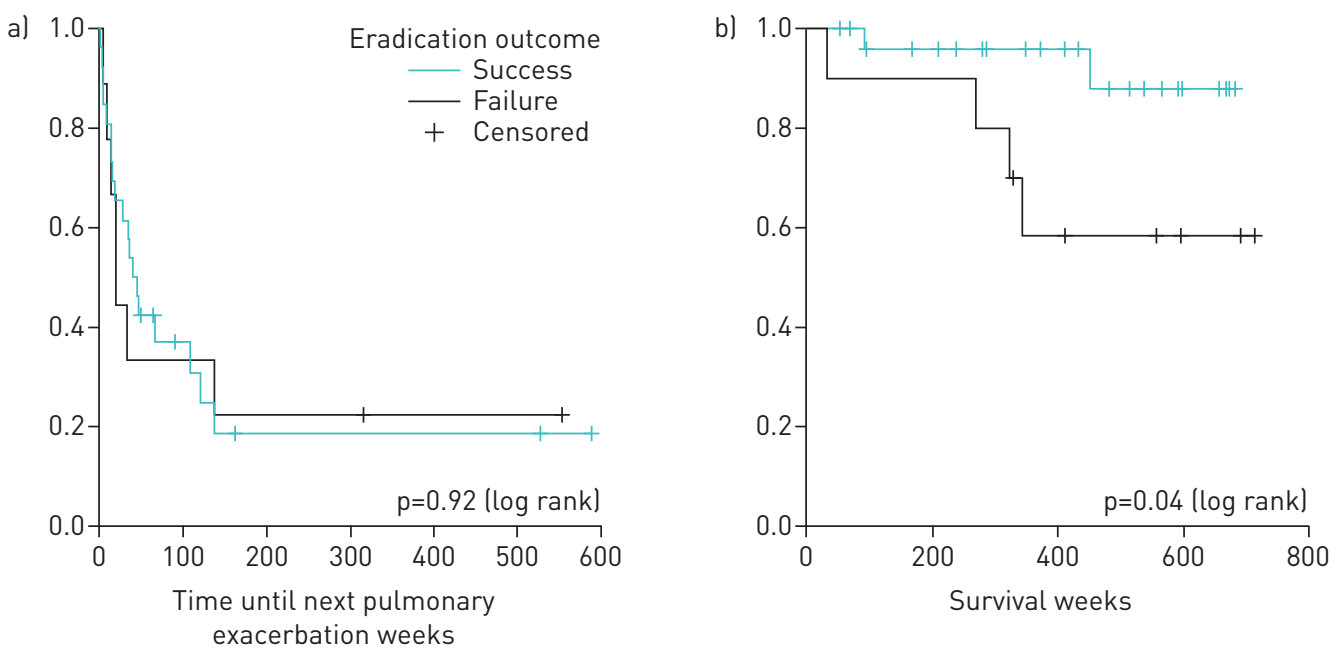

FIGURE 1 Long-term clinical outcomes. a) Time until next exacerbation after methicillin-resistant Staphylococcus aureus (MRSA) eradication and b) survival after MRSA respiratory infection.

eradication course $(n=8)$, the similar efficacy rates observed between both groups are reassuring (short course $88 \%$ versus long courses $69 \%$ ). Furthermore, these results are in accordance with a previous study [14], which also reported that protocols using longer courses of rifampicin and fusidic acid were no more effective than shorter ones. While awaiting clinical trials that would clarify this question, all these findings taken together suggest that rifampicin and fusidic acid could be prescribed for 3 weeks to maximise compliance and minimise side-effects with no compromise on efficacy.

Our study also demonstrated that successful eradication was less likely in patients who had at least one peripheral screening swab positive for MRSA. This novel finding concurs with the previous hypothesis of VANDERHELST et al. [21], who postulated that patients with negative nasal screening might be more susceptible to spontaneous eradication of MRSA without treatment. In our study, all patients were offered the same nasal/skin decolonisation protocol. Since retrospective evaluation of treatment adherence is inaccurate, efficacy of topical treatment was not assessed. Nevertheless, as suggested by studies performed in the non-CF population [22], topical decolonisation is expected to enhance eradication of MRSA carriage and reduce the likelihood of reinfection. Therefore, a holistic approach combining systemic and topical treatments with environmental advice (e.g. regular washing of bed linen and towels, avoidance of toothbrush sharing, etc.) should be employed to optimise MRSA clearance. Additionally, MRSA screening of household members, with subsequent decolonisation if positive, should also be considered.

Despite both groups of people with CF included in our study having similar clinical characteristics at the initiation of eradication treatment, the subgroup of patients who were not successfully eradicated had a worse survival rate than those who underwent successful eradication. This finding supports the results from a large cohort study [5] and underlines the clinical importance of pro-active management of new MRSA infection in order to optimise clinical prognosis for CF patients. We also observed that there was a higher proportion of F508del-homozygous patients in the eradication failure subgroup. This may bias the results of our work due to the poorer prognosis conferred by this genotype. However, both groups were otherwise similar, including FEV1 values and, when studying an adult cohort, lung function data may be more predictive of patient survival than genotype alone [23].

In this study, seven different eradication regimens were observed (table 1). Such heterogeneity of practice is partly explained by the fact that most of the study period, defined from 2000 to 2014, occurred before the publication of the first UK guidelines in May 2009, which suggested a specific protocol for MRSA eradication. Furthermore, clinical guidelines are recommendations and not mandatory lines of practice. Treatment decisions are made by the clinician on an individual basis according to the medical history of the patient (e.g. allergies and intolerances) and the antimicrobial susceptibility profile of the bacterial isolate. This inevitably leads to treatment disparity. Consequently, as previously discussed, the variety of regimens observed in this study (antibiotics and treatment duration) prevented the elaboration of strong conclusions with regards to the superiority of a specific eradication protocol.

At the Northern Ireland Regional CF Centre, normal practice is to attempt eradication of MRSA as soon as one respiratory sample for MRSA is reported. Although a second respiratory sample is routinely collected at initiation of eradication, the result does not impact on clinical management. In fact, the eradication protocol 
is continued even if MRSA is not cultured from the second respiratory sample. Amongst our patients, nine (24\%) had only one positive respiratory culture documented. However, two of these patients also had a concurrent positive peripheral screening swab (nasal, throat or perineal), resulting in seven patients with only one positive sample. Therefore, our practice results in the treatment of people who may have spontaneously cleared MRSA infection without therapy. As a result, our observed eradication success rate may be overestimated. It was previously reported that between one-third and up to half of CF patients positive for MRSA in a single respiratory sample will spontaneously clear the bacterium without specific treatment [1, 24]. Furthermore, MRSA isolates were not available for molecular typing, which prevents determination of whether eradication failure was associated with a single strain. Similarly, it was not possible to determine if patients with multiple positive samples were persistently infected with the same strain.

\section{Conclusion}

Due to its deleterious impact on morbidity and mortality in people with CF, it is widely recognised that MRSA warrants careful surveillance and pro-active management to prevent establishment of chronic respiratory infection. This important study showed that patients with positive peripheral screening for MRSA were less likely to clear their respiratory tract infection. This finding underlines the necessity to prescribe and promote adherence to topical treatment and environmental decolonisation measures in addition to systemic antibiotics in order to reduce reinfection. This study also emphasises the feasibility of MRSA eradication in new onset infections and highlights the detrimental impact on survival if eradication is not achieved. Results of ongoing and future clinical trials are awaited in order to define the optimal treatment regimen and duration for eradication of newly acquired MRSA infection in CF.

\section{Acknowledgements}

The authors would like to acknowledge L. Sherrard (Cystic Fibrosis \& Airways Microbiology Research Group, Queen's University Belfast, Belfast, UK) for the thorough editing of this manuscript.

\section{References}

1 Dasenbrook EC, Merlo CA, Diener-West M, et al. Persistent methicillin-resistant Staphylococcus aureus and rate of FEV1 decline in cystic fibrosis. Am J Respir Crit Care Med 2008; 178: 814-821.

2 Cox DW, Kelly C, Rush R, et al. The impact of MRSA infection in the airways of children with cystic fibrosis: a case-control study. Ir Med J 2011; 104: 305-308.

3 Sawicki GS, Rasouliyan L, Pasta DJ, et al. The impact of incident methicillin resistant Staphylococcus aureus detection on pulmonary function in cystic fibrosis. Pediatr Pulmonol 2008; 43: 1117-1123.

4 Vanderhelst E, De Meirleir L, Verbanck S, et al. Prevalence and impact on FEV1 decline of chronic methicillin-resistant Staphylococcus aureus (MRSA) colonization in patients with cystic fibrosis. A single-center, case control study of 165 patients. J Cyst Fibros 2012; 11: 2-7.

5 Dasenbrook EC, Checkley W, Merlo CA, et al. Association between respiratory tract methicillin-resistant Staphylococcus aureus and survival in cystic fibrosis. JAMA 2010; 303: 2386-2392.

6 Cystic Fibrosis Trust. Recommendations - eradication and treatment of MRSA. In: Antibiotic Treatment for Cystic Fibrosis. 3rd Edn. Bromley, Cystic Fibrosis Trust, 2009.

7 Döring G, Flume P, Heijerman H, et al. Treatment of lung infection in patients with cystic fibrosis: current and future strategies. J Cyst Fibros 2012; 11: 461-479.

8 Jennings MT, Boyle MP, Weaver D, et al. Eradication strategy for persistent methicillin-resistant Staphylococcus aureus infection in individuals with cystic fibrosis - the PMEP trial: study protocol for a randomized controlled trial. Trials 2014; 15: 223

9 Burdge DR, Nakielna E, Noble M. Eradication of methicillin-resistant Staphylococcus aureus from the lower respiratory tract of patients with cystic fibrosis. Can J Infect Dis 1995; 6: 97-101.

10 Doe SJ, McSorley A, Isalska B, et al. Patient segregation and aggressive antibiotic eradication therapy can control methicillin-resistant Staphylococcus aureus at large cystic fibrosis centres. J Cyst Fibros 2010; 9: 104-109.

11 Garske LA, Kidd TJ, Gan R, et al. Rifampicin and sodium fusidate reduces the frequency of methicillin-resistant Staphylococcus aureus (MRSA) isolation in adults with cystic fibrosis and chronic MRSA infection. J Hosp Infect 2004; 56: 208-214.

12 Macfarlane M, Leavy A, McCaughan J, et al. Successful decolonization of methicillin-resistant Staphylococcus aureus in paediatric patients with cystic fibrosis (CF) using a three-step protocol. J Hosp Infect 2007; 65: 231-236.

13 Vanderhelst E, De Wachter E, Willekens J, et al. Eradication of chronic methicillin-resistant Staphylococcus aureus infection in cystic fibrosis patients. An observational prospective cohort study of 11 patients. J Cyst Fibros 2013 12: $662-666$

14 Hall H, Gadhok R, Alshafi K, et al. Eradication of respiratory tract MRSA at a large adult cystic fibrosis centre Respir Med 2015; 109: 357-363.

15 Serisier DJ, Jones G, Carroll M. Eradication of pulmonary methicillin-resistant Staphylococcus aureus (MRSA) in cystic fibrosis with linezolid. J Cyst Fibros 2004; 3: 61.

16 Solis A, Brown D, Hughes J, et al. Methicillin-resistant Staphylococcus aureus in children with cystic fibrosis: an eradication protocol. Pediatr Pulmonol 2003; 36: 189-195.

17 Lo DK, Hurley MN, Muhlebach MS, et al. Interventions for the eradication of meticillin-resistant Staphylococcus aureus (MRSA) in people with cystic fibrosis. Cochrane Database Syst Rev 2015; 2: CD009650.

18 Cystic Fibrosis Trust. Methicillin-resistant Staphylococcus aureus (MRSA). (Report of the UK Cystic Fibrosis Trust Infection Control Group) Bromley, Cystic Fibrosis Trust, 2008. 
19 Liu C, Bayer A, Cosgrove SE, et al. Clinical practice guidelines by the Infectious Diseases Society of America for the treatment of methicillin-resistant Staphylococcus aureus infections in adults and children. Clin Infect Dis 2011; 52: e18-e55.

20 ESCMID Consensus concerning MRSA. Clin Microbiol Infect 2009; 15: Suppl. 7, 1-52.

21 Vanderhelst E, De Meirleir L, Verbanck S, et al. Reply to "methicillin-resistant Staphylococcus aureus". J Cyst Fibros 2013; 12: 183.

22 Ammerlaan HS, Kluytmans JA, Wertheim HF, et al. Eradication of methicillin-resistant Staphylococcus aureus carriage: a systematic review. Clin Infect Dis 2009; 48: 922-930.

23 Kerem E, Reisman J, Corey M, et al. Prediction of mortality in patients with cystic fibrosis. N Engl J Med 1992; 326: $1187-1191$.

24 Thomas SR, Gyi KM, Gaya H, et al. Methicillin-resistant Staphylococcus aureus: impact at a national cystic fibrosis centre. J Hosp Infect 1998; 40: 203-209. 